\title{
COMPARISON OF TWO TIME-STEPPING METHODS FOR COMPOSITIONAL RESERVOIR SIMULATION
}

B. R. B. Fernandes ${ }^{2}$, A. Varavei $^{3}$, F. Marcondes ${ }^{1}$, and K. Sepehrnoori ${ }^{3}$

${ }^{1}$ Department of Metallurgical Engineering and Material Science at the Federal University of Ceará, Ceará, Brazil (marcondes@ufc.br)

${ }^{2}$ Laboratory of Computational Dynamics of Fluids, LDFC, Department of Metallurgical Engineering and Material Science at the Federal University of Ceará, Ceará, Brazil

${ }^{3}$ Petroleum and Geosystems Engineering Department, The University of Texas at Austin, Texas, USA

Abstract. In this paper, a comparison of two compositional reservoir simulation formulations is presented. The first formulation is based on an implicit pressure and explicit concentration (IMPEC) procedure and the second formulation uses an implicit pressure and implicit saturation (IMPSAT) for the solution of the governing partial differential equations. Our main goal was to compare the formulations in terms of computational times for solving $2 D$ and $3 D$ compositional reservoir simulation case studies. Both single point upstream weighting and third-order schemes for discretization of the partial differential equations were implemented for the formulations. The computational results for the abovementioned formulations for several case studies involving homogeneous and heterogeneous reservoirs are presented in this paper.

Keywords: Segregated Formulation, IMPEC, Compositional Reservoir Simulation.

\section{INTRODUCTION}

Several formulations have been developed for solving the governing partial differential equations arising from modeling fluid flow for compositional simulations in porous media. In general, these formulations can be classified as Implicit Pressure Explicit Saturation (IMPEC), Implicit Pressure and Saturation (IMPSAT), or the Fully Implicit Method (FIM). The IMPEC formulation has the lowest cost in terms of computational time per time-step. However, due to the explicit calculation of concentrations, this formulation cannot use large time-steps when compared to the FIM and IMPSAT approaches. The IMPSAT method can handle larger time-steps when compared to the IMPEC approach, but it is less expensive in terms of computational time, per time-step, than the FIM approach. 
In this work, an IMPSAT formulation proposed by Watts [1] was implemented into the UTCOMP simulator. UTCOMP was developed at the Center for Petroleum and Geosystems Engineering at The University of Texas at Austin for the simulation of enhanced recovery processes. The original approach of the UTCOMP simulator is an IMPEC [2,3], multiphase/multi-component compositional equation-of-state simulator, which can handle the simulation of several enhanced oil recovery processes.

Other procedures for solving the pressure and saturation implicitly (IMPSAT) were proposed in [4] and [5]. However, the one presented here has similar advantages as the original IMPEC formulation in the UTCOMP simulator. In other words, this IMPSAT formulation solves simple sets of linear systems for both pressure and saturations, while only one flash procedure is performed per time-step. Also, for most test cases, the formulation was able to use larger time-steps than IMPEC formulation.

\section{PHYSICAL MODEL}

We now present the main equations of the Watts' [1] formulation. Watts' approach is basically an adaptation of the method proposed in [4] where the volume error constraint is added to pressure and saturation equations. In this section, we show the molar balance equations, the pressure equation, and the new saturation equations.

\subsection{Molar Balance Equation}

The molar balance equations considering the advection transport term (see [3]) is given by

$$
\frac{\partial N_{k}}{\partial t}=-V_{b} \sum_{j=1}^{N_{p}} x_{k j} \xi_{j} \nabla \cdot \vec{v}_{j}-q_{k}, \quad k=1, \ldots, N_{c}, N_{c}+1
$$

where $N_{k}$ is the moles of component $k, V_{b}$ is the bulk volume, $x_{k j}$ is the mole fraction of component $k$ in phase $j, \xi_{j}$ is the molar density, $\mathrm{v}_{j}$ is the velocity of phase $j, q_{k}$ is the molar rate of component $k$ through the well, $N_{c}$ is the number of hydrocarbon components, and $N_{c}+1$ denotes the water component.

The velocity of phase $j$ is given by

$$
\vec{v}_{j}=-\frac{k_{r j}}{\mu_{j}} \overline{\bar{K}} \cdot \vec{\nabla} \Phi_{j},
$$

where $k_{r j}$ and $\mu_{j}$ are the relative permeability and viscosity of phase $j$, respectively, $\overline{\bar{K}}$ is the absolute permeability tensor, and $\Phi_{j}$ is the hydraulic potential of phase $j$ which is defined by

$$
\Phi_{j}=P-\rho_{j} g D-P_{g r}
$$


where $P$ is the pressure of oil phase, $\rho_{j}$ is the mass density of phase $j, g$ is the gravity, $D$ is the depth, and $P_{c r j}$ is the capillary pressure between phase $j$ and $r$.

\subsection{Fluid Equilibrium Criteria}

For the closure equations, fluid phase equilibrium between the hydrocarbon phases is used. This assumption considers that the chemical potential of all phases are the same. This can be expressed in terms of the equality of the fugacities $(f)$ of the phases, as shown below:

$$
f_{i}^{o}-f_{i}^{g}=0, \quad i=1, \ldots, N_{c} .
$$

\subsection{Pressure Equation}

In this work the pressure equation used in UTCOMP simulator, for IMPEC and Watts formulations, is based on the volume constraint proposed as in [2]. The pressure equation is obtained from the equality between the formation pore volume $\left(V_{p}\right)$ and the total fluid volume $\left(V_{t}\right)$.

$$
V_{p}(P)=V_{t}\left(P, N_{1}, \ldots, N_{N_{c}}, N_{N_{C}+1}\right)
$$

Taking the derivative of Eq. (5) with respect to time, and applying the chain rule to the right-hand side, we obtain:

$$
\left(\frac{\partial V_{p}}{\partial P}\right) \frac{\partial P}{\partial t}=\left(\frac{\partial V_{t}}{\partial P}\right)_{N} \frac{\partial P}{\partial t}+\sum_{k=1}^{N_{c}+1}\left(\frac{\partial V_{t}}{\partial N_{k}}\right)_{P, N_{S}(s \neq k)} \frac{\partial N_{k}}{\partial t}
$$

The derivative of total fluid volume with respect to pressure is equal to the total fluid compressibility multiplied by the total fluid volume, and the derivative of total fluid volume with respect to the number of moles of each component is equal to the partial molar volume derivative.

The pore volume is related to the porosity $(\phi)$ and the bulk volume $\left(V_{b}\right)$, by

$$
V_{p}=V_{b} \phi
$$

and

$$
\phi=\phi_{0}\left(1+C_{f}\left(P-P_{f}\right)\right)
$$

where $\phi_{o}$ is the porosity at the reference pressure $\left(P_{f}\right)$, and $C_{f}$ is the rock compressibility.

Substituting Eqs. (7) and (8) into Eq. (6), we obtain:

$$
V_{b} \phi_{0} C_{f} \frac{\partial P}{\partial t}=\left(\frac{\partial V_{t}}{\partial P}\right)_{N} \frac{\partial P}{\partial t}+\sum_{k=1}^{N_{c}+1}\left(\frac{\partial V_{t}}{\partial N_{k}}\right)_{P, N_{S}(s \neq k)} \frac{\partial N_{k}}{\partial t}
$$


The total volume derivative with respect to each molar component can be rewritten as

$$
\bar{V}_{t k}=\left(\frac{\partial V_{t}}{\partial N_{k}}\right)_{P, N_{s}(s \neq k)}, \quad k=1, \ldots, N_{c}, N_{c}+1 .
$$

Substituting Eqs. (1), (2) and (10) into Eq.(9), one obtains the final equation for pressure:

$$
V_{b} \phi_{0} C_{f} \frac{\partial P}{\partial t}=\left(\frac{\partial V_{t}}{\partial P}\right)_{N} \frac{\partial P}{\partial t}+V_{b} \sum_{k=1}^{N_{c}+1} \bar{V}_{t k}\left[\sum_{j=1}^{N_{p}} x_{k j} \xi_{j} \nabla \cdot\left(\frac{k_{r j}}{\mu_{j}} \overline{\bar{K}} \cdot \vec{\nabla} \Phi_{j}\right)+\frac{q_{k}}{V_{b}}\right]
$$

\subsection{Saturation Equation}

The saturation equation is solved implicitly only for the IMPSAT formulation. The approach used here is described in [1]. By definition, the saturation of phase $\ell\left(S_{\ell}\right)$ is given by

$$
S_{\ell}=\frac{V_{\ell}}{V_{p}}
$$

where $V_{\ell}$ is the volume of phase $\ell$ which is a function of pressure and number of moles. Equation (12) can be written as

$$
S_{\ell} V_{p}(P)=V_{\ell}\left(P, N_{1}, \ldots, N_{N_{C}}, N_{N_{C}+1}\right)
$$

Taking the derivative of Eq. (13) with respect to time and applying the chain rule on the right-hand side, we obtain:

$$
\frac{\partial}{\partial t}\left(S_{\ell} V_{p}\right)=\left(\frac{\partial V_{\ell}}{\partial P}\right)_{N} \frac{\partial P}{\partial t}+\sum_{k=1}^{N_{c}+1} \bar{V}_{\ell k} \frac{\partial N_{k}}{\partial t}
$$

Substituting Eq. (1) into Eq. (14) results in

$$
\frac{\partial}{\partial t}\left(S_{\ell} V_{p}\right)=\left(\frac{\partial V_{\ell}}{\partial P}\right)_{N} \frac{\partial P}{\partial t}+V_{b} \sum_{k=1}^{N_{c}+1} \bar{V}_{\ell k}\left(\sum_{j=1}^{N_{p}} x_{k j} \xi_{j} \nabla \cdot \vec{v}_{j}+\frac{q_{k}}{V_{b}}\right) .
$$

Comparing Eqs. (11) and (15), we can observe that saturations and pressure equations have the same format. As suggested in references [1] and [4], the phase velocity that is needed in Eq. (15) should be evaluated as a function of the total velocity. According to these authors, if this approach is performed, the segregated solution of pressure and saturations will be equivalent to a fully implicit procedure in terms of pressure and saturation. Next we present the expression for the total velocity using the ideas given in [1], but now considering a full permeability tensor. Considering a three-phase flow, the total velocity $\left(\mathrm{v}_{\mathrm{t}}\right)$ is given by

$$
\overrightarrow{v_{t}}=\overrightarrow{v_{w}}+\overrightarrow{v_{o}}+\overrightarrow{v_{g}},
$$


where the subscripts $w, o, g$ denote water, oil and gas, respectively.

The velocity of phase $j$ defined in Eq. (2), can be rewritten as

$$
\overrightarrow{v_{j}}=\lambda_{j} \overline{\bar{K}} \cdot\left(\vec{\nabla} P-\rho_{j} g \vec{\nabla} D+\vec{\nabla} P_{c j o}\right),
$$

where the phase mobility $\left(\lambda_{j}\right)$ is defined as

$$
\lambda_{j}=\frac{k_{r j}}{\mu_{j}}
$$

Equation (17) can be rearranged as

$$
\overline{\bar{K}} \cdot \vec{\nabla} P=-\frac{\overrightarrow{v_{j}}}{\lambda_{j}}+\rho_{j} g \overline{\bar{K}} \cdot \vec{\nabla} D-\overline{\bar{K}} \cdot \vec{\nabla} P_{c j o}
$$

Subtracting Eq. (19) with $j=w$ from Eq. (19) with $j=o$, one obtains:

$$
-\frac{\overrightarrow{v_{o}}}{\lambda_{o}}+\frac{\overrightarrow{v_{w}}}{\lambda_{w}}+\left(\rho_{o} g \overline{\bar{K}} \cdot \vec{\nabla} D-\rho_{w} g \overline{\bar{K}} \cdot \vec{\nabla} D-\overline{\bar{K}} \cdot \vec{\nabla} P_{c o o}+\overline{\bar{K}} \cdot \vec{\nabla} P_{c w o}\right)=0
$$

where

$$
\overrightarrow{v_{w}}=\overrightarrow{v_{t}}-\overrightarrow{v_{o}}-\overrightarrow{v_{g}} .
$$

Substituting Eq. (21) into Eq. (20), we obtain:

$$
-\frac{\overrightarrow{v_{o}}}{\lambda_{o}}+\frac{\overrightarrow{v_{t}}-\overrightarrow{v_{o}}-\overrightarrow{v_{g}}}{\lambda_{w}}+\left(\rho_{o} g \overline{\bar{K}} \cdot \vec{\nabla} D-\rho_{w} g \overline{\bar{K}} \cdot \vec{\nabla} D-\overline{\bar{K}} \cdot \vec{\nabla} P_{c o o}+\overline{\bar{K}} \cdot \vec{\nabla} P_{c w o}\right)=0 .
$$

In order to use only the oil phase velocity in Eq. (22), the gas velocity must be replaced. To replace the gas velocity, we can subtract Eq. (19) with $j=o$ from Eq. (19) with $j=g$.

$$
\overrightarrow{v_{g}}=\lambda_{g} \frac{\overrightarrow{v_{o}}}{\lambda_{o}}+\lambda_{g}\left(\rho_{g} g \overline{\bar{K}} \cdot \vec{\nabla} D-\rho_{o} g \overline{\bar{K}} \cdot \vec{\nabla} D-\overline{\bar{K}} \cdot \vec{\nabla} P_{c g o}+\overline{\bar{K}} \cdot \vec{\nabla} P_{c o o}\right)
$$

Substituting Eq. (23) into Eq. (22) results in

$$
\begin{aligned}
0= & -\frac{\overrightarrow{v_{o}}}{\lambda_{o}}+\frac{\overrightarrow{v_{t}}-\overrightarrow{v_{o}}}{\lambda_{w}}-\frac{\lambda_{g}}{\lambda_{w}} \frac{\overrightarrow{v_{o}}}{\lambda_{o}}-\frac{\lambda_{g}}{\lambda_{w}}\left(\rho_{g} g \overline{\bar{K}} \cdot \vec{\nabla} D-\rho_{o} g \overline{\bar{K}} \cdot \vec{\nabla} D-\overline{\bar{K}} \cdot \vec{\nabla} P_{c g o}+\overline{\bar{K}} \cdot \vec{\nabla} P_{c o o}\right), \\
& +\left(\rho_{o} g \overline{\bar{K}} \cdot \vec{\nabla} D-\rho_{w} g \overline{\bar{K}} \cdot \vec{\nabla} D-\overline{\bar{K}} \cdot \vec{\nabla} P_{c o o}+\overline{\bar{K}} \cdot \vec{\nabla} P_{c w o}\right)
\end{aligned}
$$

which after some algebraic manipulation can be written as 


$$
\begin{aligned}
\overrightarrow{v_{o}}= & \frac{\lambda_{o}}{\lambda_{w}+\lambda_{o}+\lambda_{g}} \vec{v}_{t}-\frac{\lambda_{o} \lambda_{g}}{\lambda_{w}+\lambda_{o}+\lambda_{g}}\left(\rho_{g} g \overline{\bar{K}} \cdot \vec{\nabla} D-\rho_{o} g \overline{\bar{K}} \cdot \vec{\nabla} D-\overline{\bar{K}} \cdot \vec{\nabla} P_{c g o}+\overline{\bar{K}} \cdot \vec{\nabla} P_{c o o}\right) . \\
& +\frac{\lambda_{o} \lambda_{w}}{\lambda_{w}+\lambda_{o}+\lambda_{g}}\left(\rho_{o} g \overline{\bar{K}} \cdot \vec{\nabla} D-\rho_{w} g \overline{\bar{K}} \cdot \vec{\nabla} D-\overline{\bar{K}} \cdot \vec{\nabla} P_{c o o}+\overline{\bar{K}} \cdot \vec{\nabla} P_{c w o}\right)
\end{aligned}
$$

Using the oil phase velocity presented above, and performing a final algebraic manipulation, the velocity of phase $j$ is defined by

$$
\overrightarrow{v_{j}}=f_{j}\left(\overrightarrow{v_{t}}+\sum_{m=1}^{N o} \lambda_{m} \overline{\bar{K}} \cdot\left(g \rho_{j} \vec{\nabla} D-g \rho_{m} \vec{\nabla} D-\vec{\nabla} P_{c m o}+\vec{\nabla} P_{c j o}\right)\right)
$$

where $f_{j}$ the fractional flow of phase $j\left(f_{j}\right)$ is defined by

$$
f_{j}=\frac{\lambda_{j}}{\sum_{m=1}^{N p} \lambda_{m}} .
$$

The total velocity given by Eq. (16) can be rewritten as

$$
\vec{v}_{t}=-\sum_{j=1}^{N p} \lambda_{j} \overline{\bar{K}} \cdot\left(\vec{\nabla} P+\vec{\nabla} P_{c j o}-g \rho_{j} \vec{\nabla} D\right)
$$

Substituting Eq. (26) into (15) yields

$$
\begin{aligned}
& \frac{\partial}{\partial t}\left(S_{\ell} V_{p}\right)=\left(\frac{\partial V_{\ell}}{\partial P}\right)_{N} \frac{\partial P}{\partial t} \\
& \quad+V_{b} \sum_{k=1}^{N_{c}+1} \bar{V}_{\ell k}\left(\sum_{j=1}^{N_{p}} x_{k j} \xi_{j} \nabla \cdot\left[f_{j}\left(\vec{V}_{t}+\sum_{m=1}^{N p} \lambda_{m} K\left(g \rho_{j} \vec{\nabla} D-g \rho_{m} \vec{\nabla} D-\vec{\nabla} P_{c m o}+\vec{\nabla} P_{c j o}\right)\right)\right]+\frac{q_{k}}{V_{b}}\right)
\end{aligned}
$$

Equation (29) is the final saturation equation in terms of total velocity. The next section is devoted to show the numerical discretization applied to the above equation.

\section{APROXIMATE EQUATION}

The discretization for the pressure and molar blance equations can be found in [3] and will not be treated in this paper.

In order to obtain an approximate equation for the saturation of phase $\ell$, we will integrate Eq. (29) to the control volume of Fig. 1 and time. 


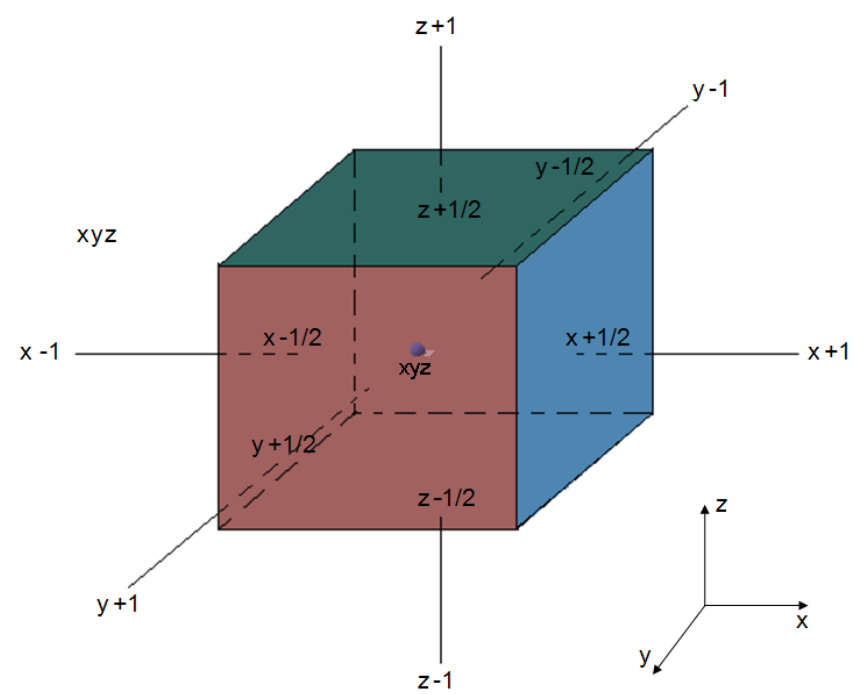

Figure 1. Control volume.

The final form of the saturation equation is shown below, for a one-dimensional control volume, given by

$$
\begin{aligned}
& S_{\ell, x y z}^{n+1} V_{p, x y z}^{n+1}-S_{\ell, x y z}^{n} V_{p, x y z}^{n}-\left(\frac{\partial V_{\ell}^{n}}{\partial P}\right)_{N}\left(P_{x y z}^{n+1}-P_{x y z}^{n}\right)+\left(S_{\ell, x y z}^{n} V_{p, x y z}^{n}-\frac{n_{\ell, x y z}^{n}}{\xi_{\ell, x y z}^{n}}\right)=\Delta t \sum_{k=1}^{N_{c}+1} \bar{V}_{\ell k, x y z}^{n} q_{k, x y z}^{n+1} \\
& +\left.\Delta t \sum_{k=1}^{N_{c}+1} \bar{V}_{\ell k, x y z}^{n} \sum_{j=1}^{N_{p}}\left(x_{k j}^{n} \xi_{j}^{n}\right)\right|_{x+1 / 2} \frac{\left.\lambda_{j}^{n+1}\right|_{x+1 / 2}}{\left.\sum_{m=1}^{N_{p}} \lambda_{m}^{n+1}\right|_{x+1 / 2}}\left\{\left.u_{t}^{n+1}\right|_{x+1 / 2}\right. \\
& +\left.\left.T_{x}\right|_{x+1 / 2} \sum_{m=1}^{N p} \lambda_{m}^{n+1}\right|_{x+1 / 2}\left[\left.g \rho_{j}^{n}\right|_{x+1 / 2}\left(D_{x+1}-D_{x y z}\right)-\left.g \rho_{m}^{n}\right|_{x+1 / 2}\left(D_{x+1}-D_{x y z}\right)\right. \\
& \left.\left.+\left(P_{c m o, x+1}^{n+1}-P_{c m o, x y z}^{n+1}\right)-\left(P_{c j o, x+1}^{n+1}-P_{c j o, x y z}^{n+1}\right)\right]\right\} \\
& -\left.\Delta t \sum_{k=1}^{N_{c}+1} \bar{V}_{\ell k, x y z}^{n} \sum_{j=1}^{N_{p}}\left(x_{k j}^{n} \xi_{j}^{n}\right)\right|_{x-1 / 2} \frac{\left.\left.\lambda_{j}^{n+1}\right|_{x-1 / 2} ^{N_{p}} \sum_{m=1}^{n+1}\right|_{x-1 / 2} ^{n+1}}{n_{t}}\left\{\left.u_{t}^{n+1}\right|_{x-1 / 2}\right. \\
& +\left.\sum_{m=1}^{N p} \lambda_{m}^{n+1}\right|_{x-1 / 2}\left[\left.g \rho_{j}^{n}\right|_{x-1 / 2}\left(D_{x y z}-D_{x-1}\right)-\left.g \rho_{m}^{n}\right|_{x+1 / 2}\left(D_{x y z}-D_{x-1}\right)\right. \\
& \left.\left.+\left(P_{c m o, x y z}^{n+1}-P_{c m o, x-1}^{n+1}\right)-\left(P_{c j o, x y z}^{n+1}-P_{c j o, x-1}^{n+1}\right)\right]\right\}
\end{aligned}
$$

where

$$
\left.T_{x}\right|_{x+1 / 2}=\frac{2 \Delta y \Delta z}{\left.\frac{\Delta x}{K_{x}}\right|_{x}+\left.\frac{\Delta x}{K_{x}}\right|_{x+1}} .
$$

Equation (30) is solved using the Newton-Raphson method.

Figure 2 shows the flow chart of the procedure used in UTCOMP simulator for the implementation of the Watts' formulation. As we can see in the figure, first pressure is evaluated, then there is an iterative procedure to evaluate the saturations. 


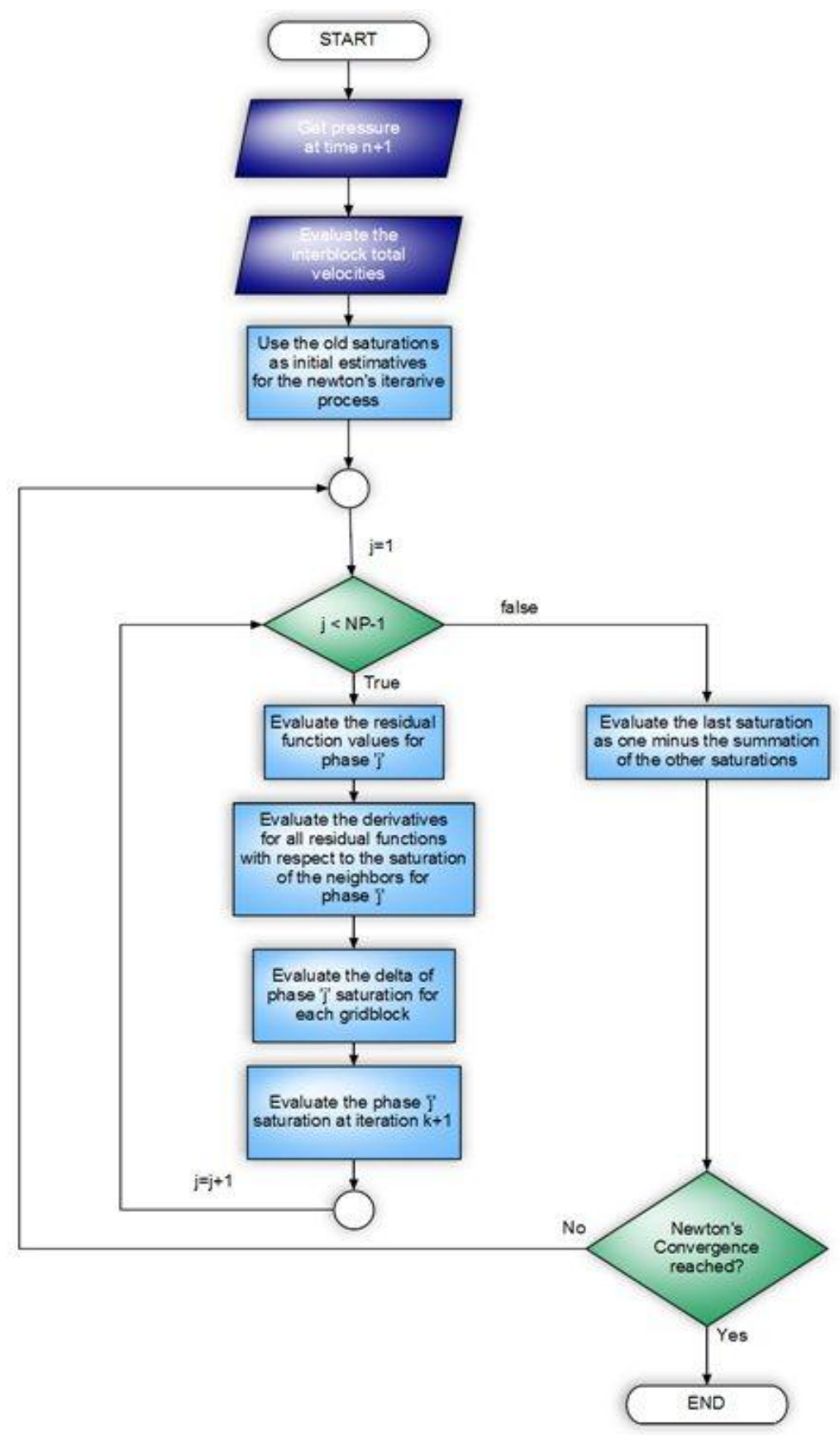

Figure 2. Flow chart for Watts' formulation.

A third-order total variation diminishing (TVD) method (see [3] and [6]) was implemented for solving the saturation equations. The relative permeabilities are treated semiimplicitly using the idea presented in [7], in which the higher order terms are considered only in the independent term of the linear system.

\section{RESULTS AND DISCUSSION}

In this section, we investigate the numerical solution as well as the performance in terms of CPU time of the Watts' formulation implemented in this work. The comparison 
studies were carried out by setting the maximum allowable time-steps for various cases to values such that comparable non-oscillatory results were obtained for both formulations.

The first case investigated is a $\mathrm{CO}_{2}$ injection in an isotropic and heterogeneous reservoir. All reservoir data are shown in Table 1.

Table 1: Reservoir data - Case 1.

\begin{tabular}{ccc}
\hline Property & Value & Unit \\
\hline Length & 500 & $\mathrm{ft}$ \\
Width & 1000 & $\mathrm{ft}$ \\
Height & 20 & $\mathrm{ft}$ \\
First Layer Depth & 2600 & $\mathrm{ft}$ \\
Porosity & 0.25 & \\
Relative Permeability Model & Corey & \\
Initial Water Saturation & 0.25 & $\mathrm{psia}$ \\
Initial Pressure & 1100 & $\mathrm{mD}$ \\
Permeability in Z direction & 10 & ${ }^{\circ} \mathrm{F}$ \\
Formation Temperature & 105 & $\mathrm{psia}$ \\
Injector BHP & 1250 & $\mathrm{psia}$ \\
Producer BHP & 1100 & \\
Grid & $20 \times 40 \mathrm{x} 1$ &
\end{tabular}

The components, initial composition and fluid injection composition are shown in Table 2 .

Table 2: Component data - Case 1.

\begin{tabular}{ccc}
\hline Component & Initial Reservoir Composition & Injection Fluid Composition \\
\hline $\mathrm{CO} 2$ & 0.0337 & 0.95 \\
$\mathrm{C} 1$ & 0.0861 & 0.04999 \\
$\mathrm{C} 2-3$ & 0.1503 & 0.000002 \\
$\mathrm{C} 4-6$ & 0.1671 & 0.000002 \\
$\mathrm{C} 7-15$ & 0.3304 & 0.000002 \\
$\mathrm{C} 16-27$ & 0.1611 & 0.000002 \\
$\mathrm{C} 28$ & 0.0713 & 0.000002 \\
\hline
\end{tabular}

The absolute permeability field in the $\mathrm{x}$ and $\mathrm{y}$ directions are shown in Fig. 3. In order to better visualize the whole variation of the permeability field, Figs. $3 b$ and $3 c$ show two different zooms of the whole scale presented in Fig. 3a. 


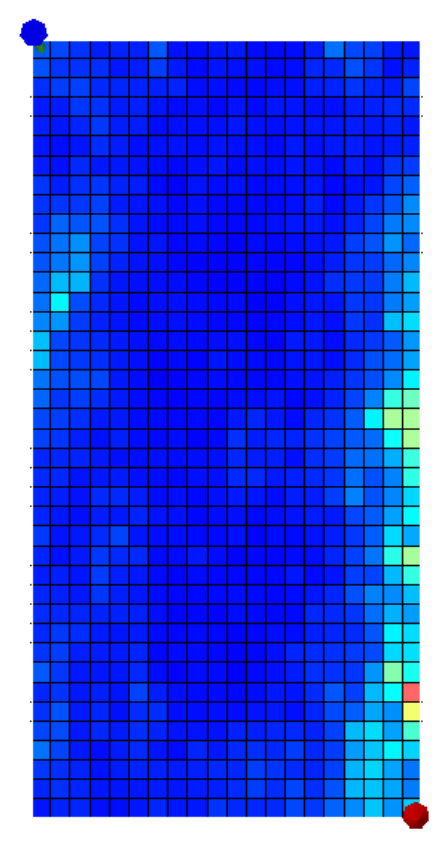

Permeability I

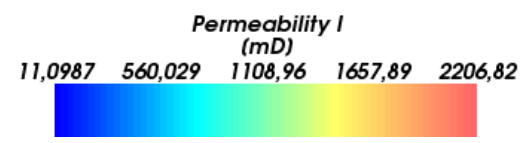

(a)

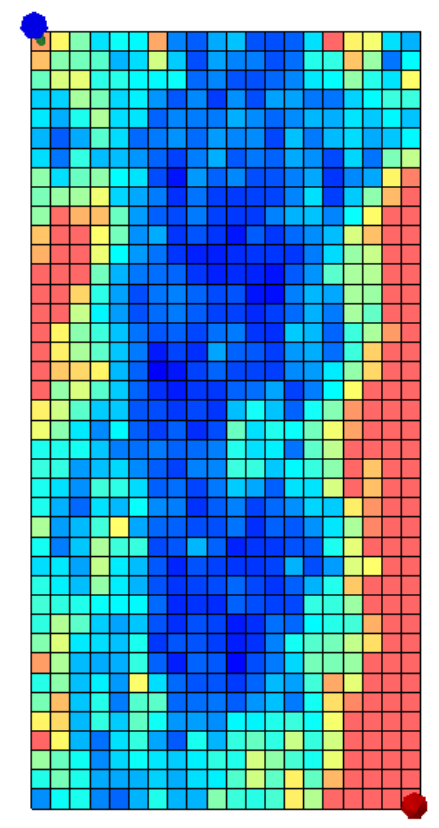

Permeability 1

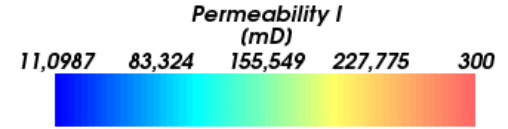

(b)
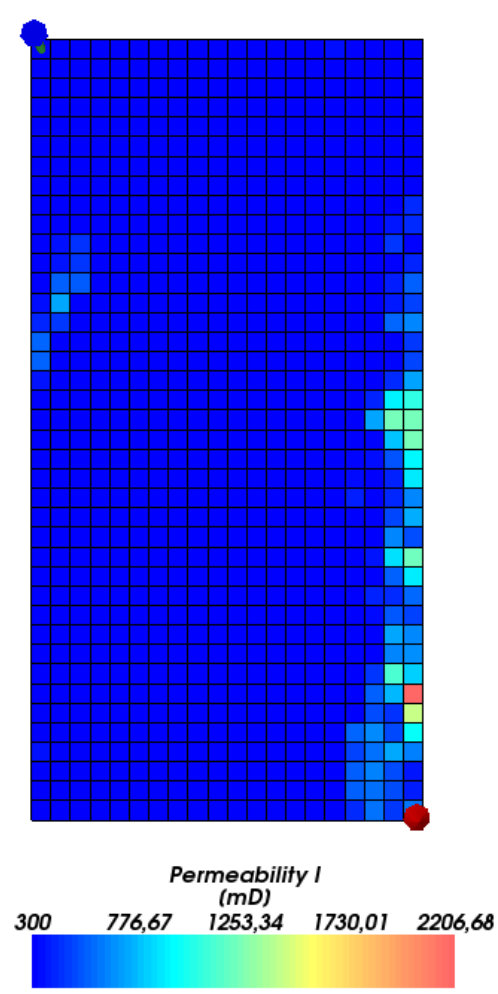

(c)

Figure 3: Permeability in the $\mathrm{x}$ and $\mathrm{y}$ direction.

We compare the results of the Watts' formulation with the original IMPEC formulation of the UTCOMP simulator. The results in terms of oil and gas production are presented in Figs. 4 and 5, respectively. From these figures, we can observe a good agreement between the original IMPEC formulation and the Watts' formulation implemented in the UTCOMP simulator.

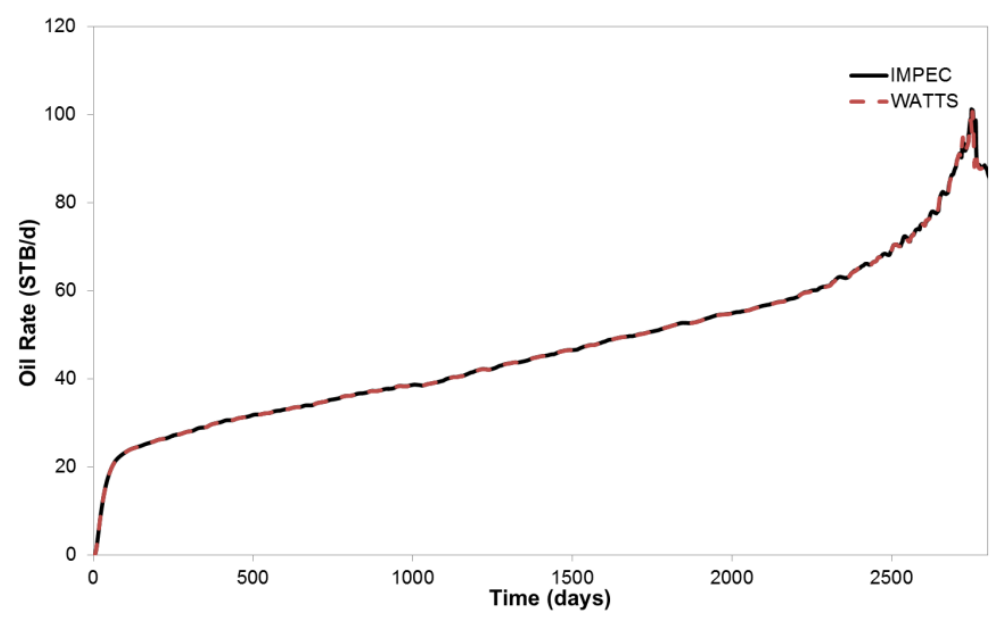

Figure 4: Oil rate - Case 1. 


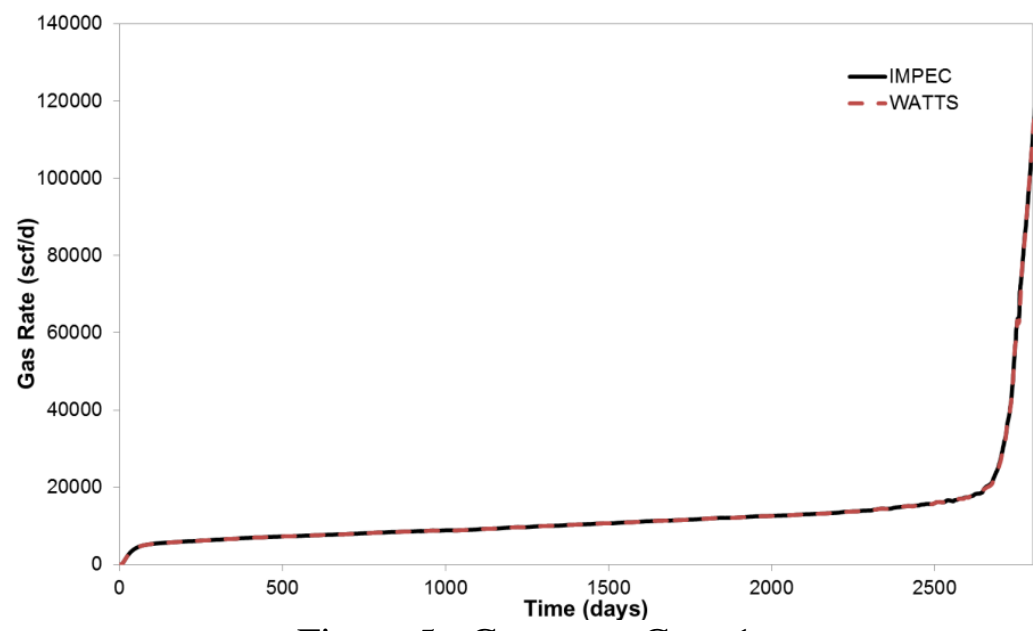

Figure 5: Gas rate - Case 1.

The results for the second liquid front at 1844 days obtained with the IMPEC and Watts' formulation is compared in Figs. 6. As can be seen in this figure, a good agreement at the two fronts is verified.

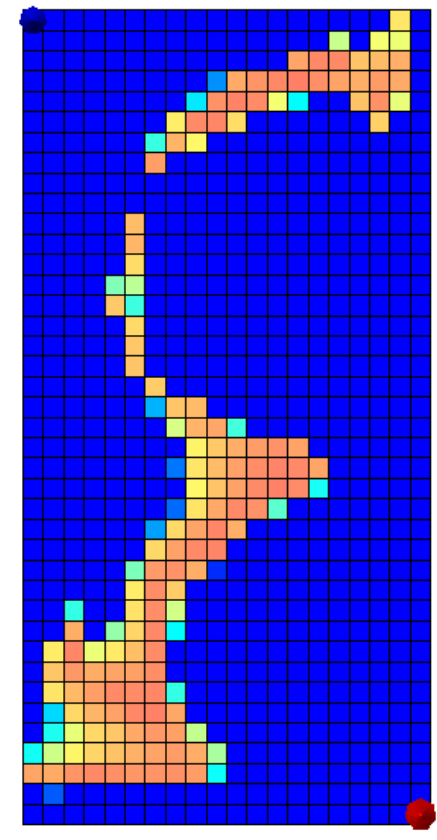

SATURATIONS OF PHASE (2ND HC) $\begin{array}{lllll}0 & 0.0855946 & 0.171189 & 0.256784 & 0.342378\end{array}$

(a)

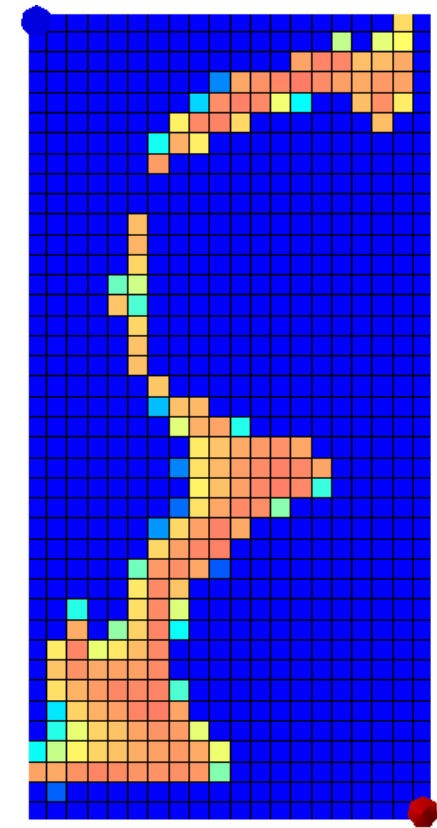

SATURATIONS OF PHASE (2ND HC)

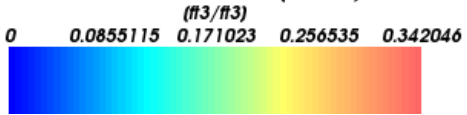

(b)

Figure 6: Second liquid front at 1844 days. a) IMPEC; b) Watts.

The time-steps used by the original UTCOMP approach (IMPEC) and the new approach (Watts) are shown in Fig. 7. 


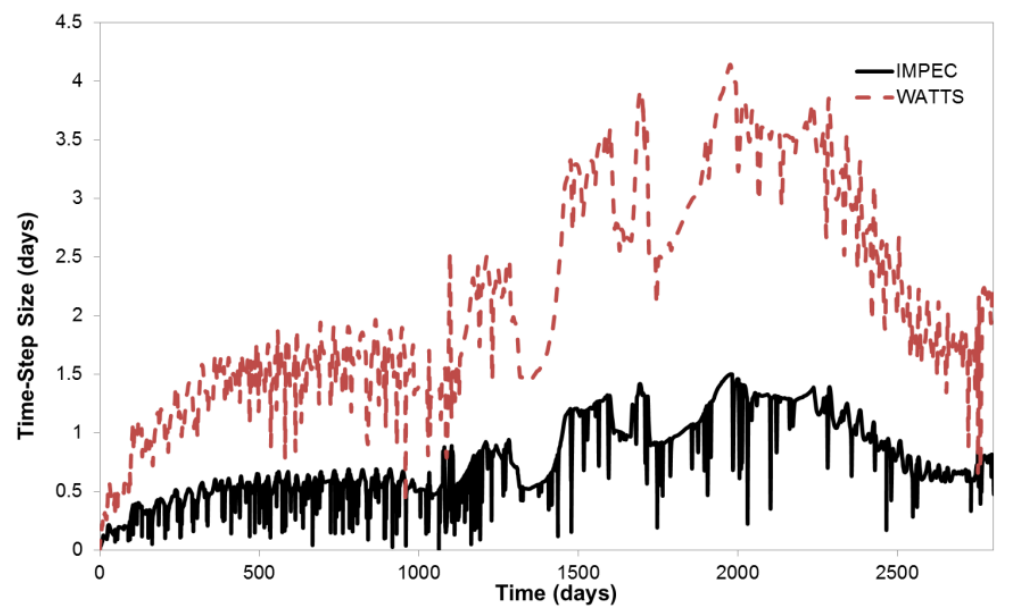

Figure 7: Time-step - Case 1.

As we can observe in Fig. 7, the time-steps used by the Watts' formulation were larger than those of the IMPEC formulation for the entire simulation. Due to the computational time for the solution of the linear system for the saturations, the Watts' formulation is more expensive per time-step. However, the large time-steps used by the Watts' formulation allowed this formulation to be less expensive in terms of CPU time than that of the IMPEC formulation. This fact can be seen in Table 3 that shows the CPU time of both formulations. From this table, we observe that Watts' formulation was about two times faster than the IMPEC formulation.

Table 3: CPU time comparison - Case 1.

\begin{tabular}{ccc}
\hline Formulation & CPU time & Speed-up ratio \\
\hline IMPEC & 467.58 & 1 \\
Watts & 225.21 & 2.08 \\
\hline
\end{tabular}

Case 2 is similar to Case 1, but we replaced the upwind function by a third-order TVD interpolation function. Figures 8 and 9 show the volumetric rate of oil and gas, respectively, and Fig. 10 presents the second liquid saturation front at 1844 days. Once again, the Watts' formulation results are in good agreement with the IMPEC formulation.

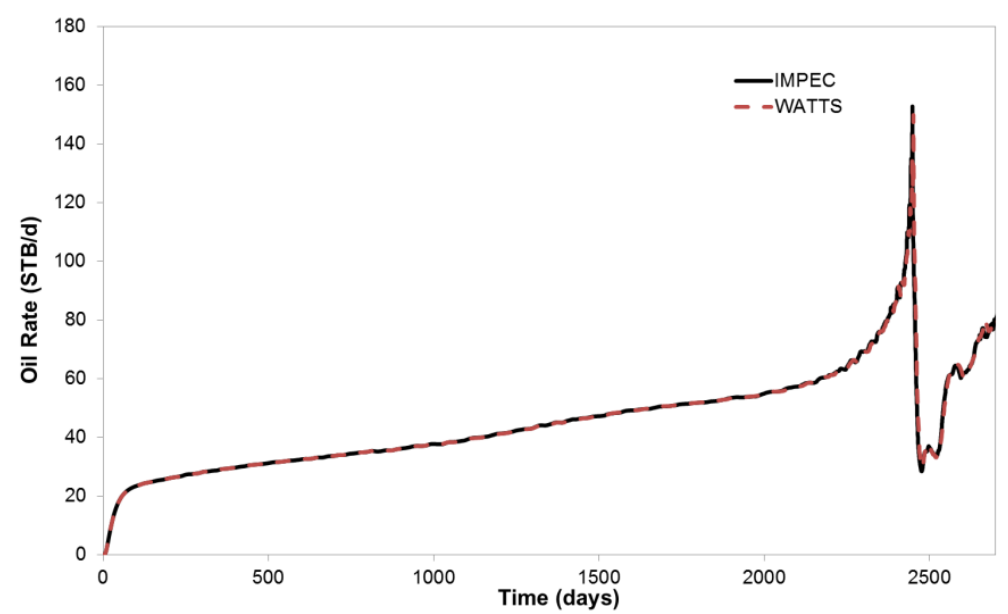

Figure 8: Oil volumetric rate - Case 2. 


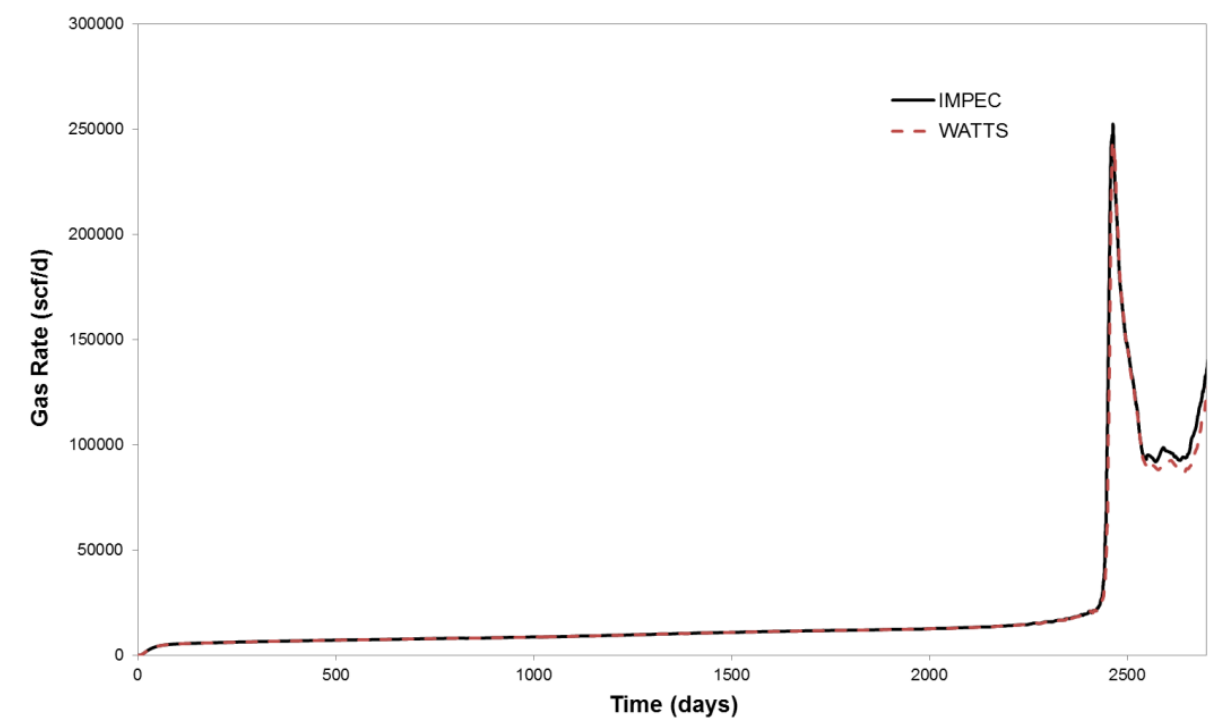

Figure 9: Gas volumetric rate - Case 2.

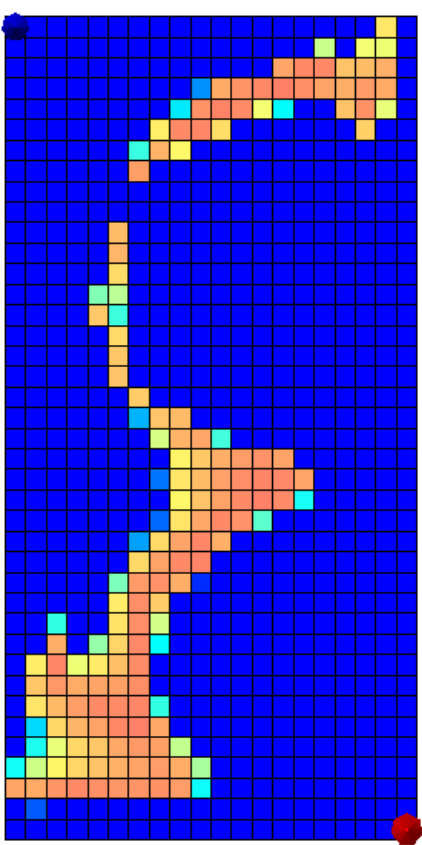

SATURATIONS OF PHASE (2ND HC) $\begin{array}{lllll}0.0855946 & 0.171189 & 0.256784 & 0.342378\end{array}$

(a)

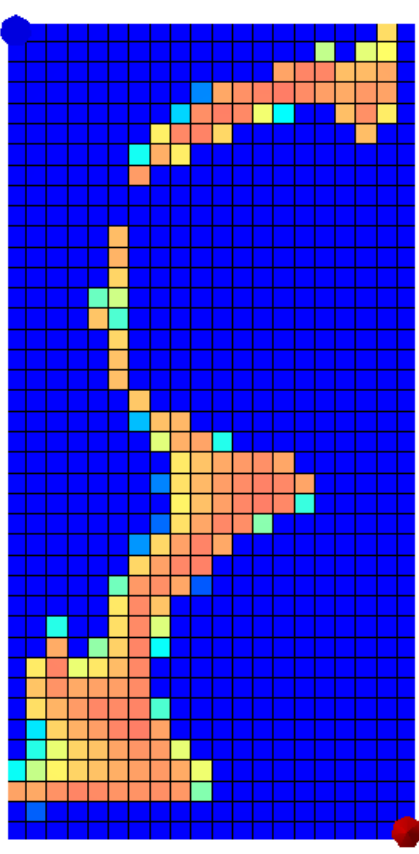

SATURATIONS OF PHASE (2ND HC) 0.08551150 .171023

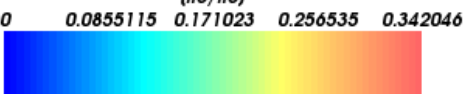

(b)

Figure 10: Second liquid front at 1844 days. a) IMPEC; b) Watts.

The time-steps used in this case study for the original UTCOMP approach (IMPEC) and the new approach (Watts) are shown in Fig. 11. Although the time-step pattern shown in Fig. 11 is different from that shown in Fig. 7 using the upwind scheme, approximately the same speed-up ratio of the Watts' compared to the IMPEC formulation for Case 1 was obtained as shown in Table 4. 


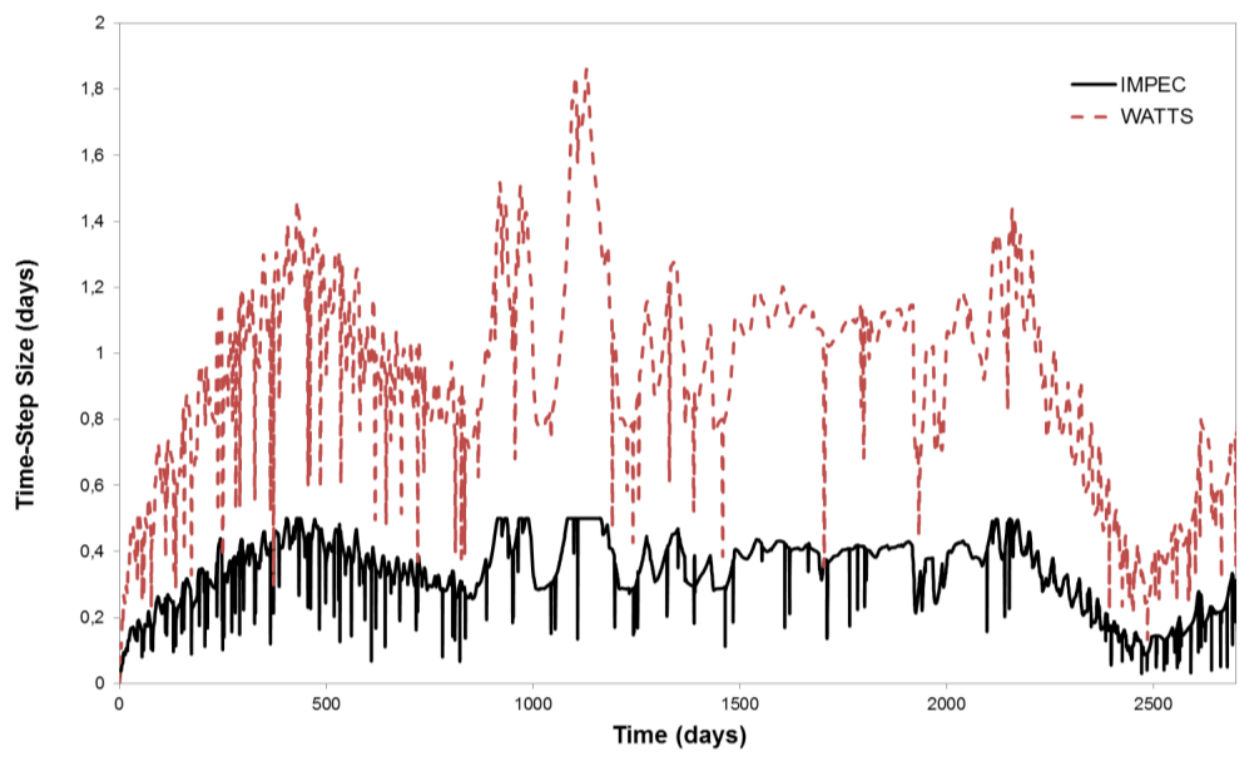

Figure 11: Time-step comparison - Case 2.

Table 4: CPU time comparison - Case 2.

\begin{tabular}{ccc}
\hline Formulation & CPU time & Speed-up ratio \\
\hline IMPEC & 980.95 & 1 \\
Watts & 482.44 & 2.03 \\
\hline
\end{tabular}

Case 3 refers to a WAG (water-alternating gas) process in a quarter-of-five-spot configuration. For this case, we start injecting $\mathrm{CO}_{2}$, and then water for one hundred days. Then, the injector well is shut in. Each fluid is injected at a fixed pressure over the course of ten days. Tables 5 and 6 present the reservoir fluid properties employed for this case.

Table 5: Reservoir data - Case 3.

\begin{tabular}{ccc}
\hline Property & Value & Unit \\
\hline Length & 480 & $\mathrm{ft}$ \\
Width & 480 & $\mathrm{ft}$ \\
Height & 47.5 & $\mathrm{ft}$ \\
First Layer Depth & 0 & $\mathrm{ft}$ \\
Porosity & 0.163 & \\
Relative Permeability Model & Baker & \\
Initial Water Saturation & 0.65 & $\mathrm{psia}$ \\
Initial Pressure & 1400 & $\mathrm{mD}$ \\
Permeability in the x and y directions & 200 & $\mathrm{mD}$ \\
Permeability in the z direction & 100 & ${ }^{\circ} \mathrm{F}$ \\
Formation Temperature & 140 & $\mathrm{psia}$ \\
Injector BHP & 1450 & $\mathrm{psia}$ \\
Producer BHP & 1000 & \\
Grid & $100 \mathrm{x} 100 \mathrm{x} 1$ &
\end{tabular}


Table 6: Component data - Case 3.

\begin{tabular}{ccc}
\hline Component & Initial Reservoir Composition & Injection Fluid Composition \\
\hline $\mathrm{CO} 2$ & 0.0077 & 1 \\
$\mathrm{C} 1$ & 0.2025 & \\
$\mathrm{C} 2-3$ & 0.1180 & \\
$\mathrm{C} 4-6$ & 0.1484 & \\
$\mathrm{C} 7-15$ & 0.2863 & \\
$\mathrm{C} 16-27$ & 0.1490 & \\
$\mathrm{C} 28$ & 0.0881 & \\
\hline
\end{tabular}

For this case, the upwind scheme was used to obtain the solution for both formulations. Figures 12 and 13 present the oil and gas volumetric rates, respectively, and Fig. 14 shows the oil saturation front at 100 days for a 100x100 grid. From Figs. 12 through 14 , we observe again that the results of both formulations are in good agreement.

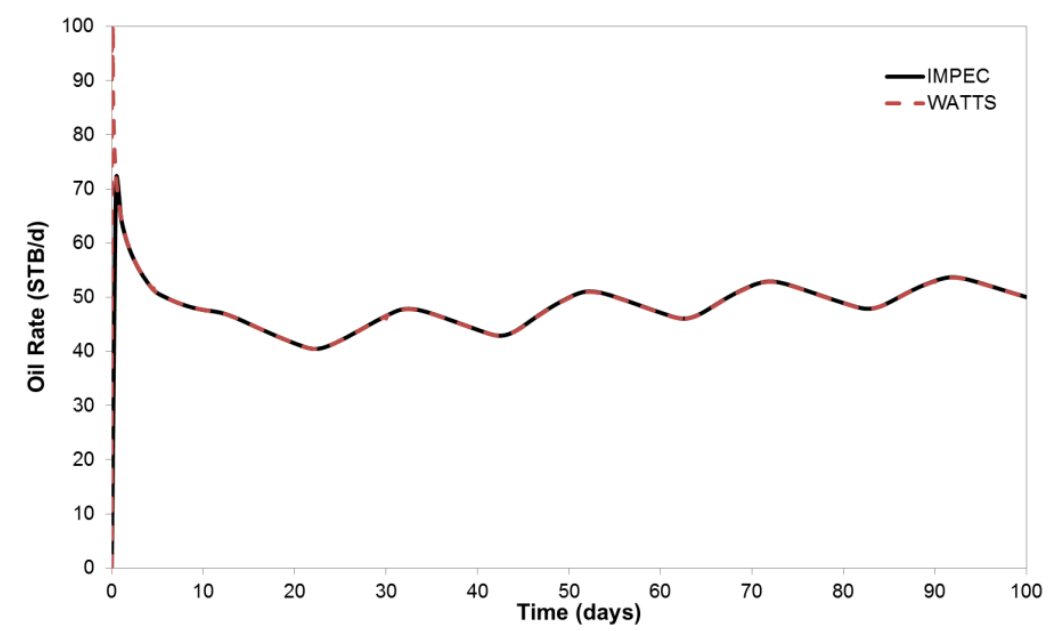

Figure 12: Oil volumetric rate - Case 3.

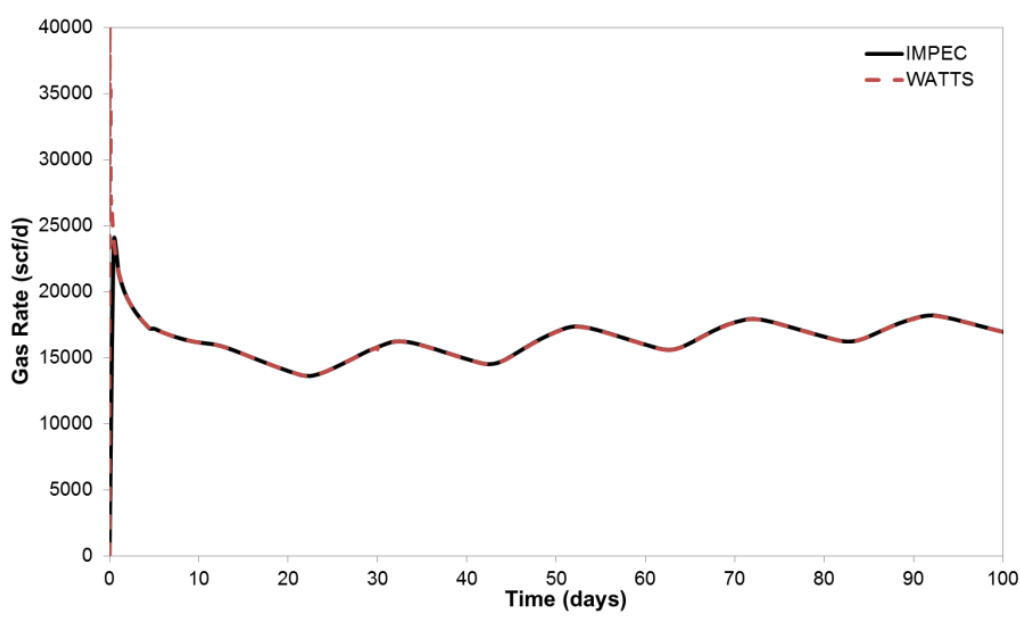

Figure 13: Gas volumetric rate - Case 3. 


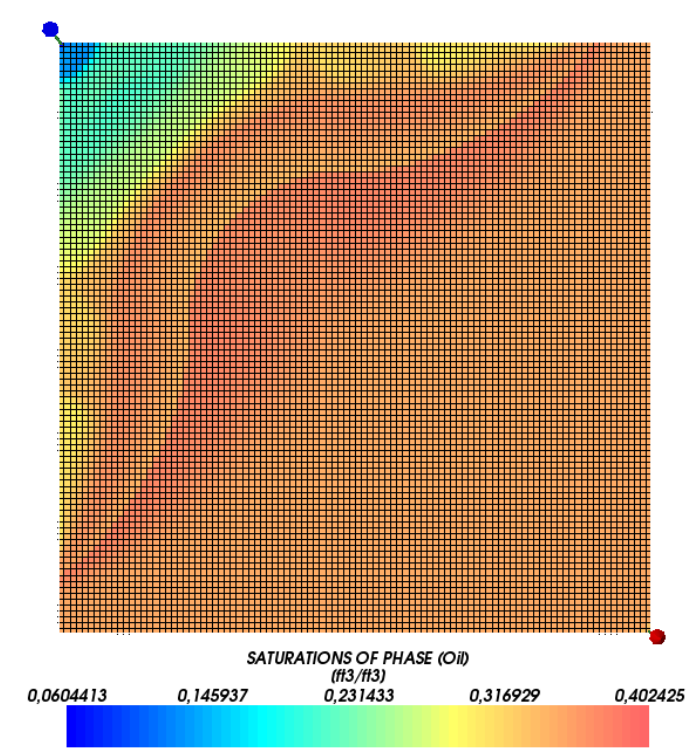

(a)

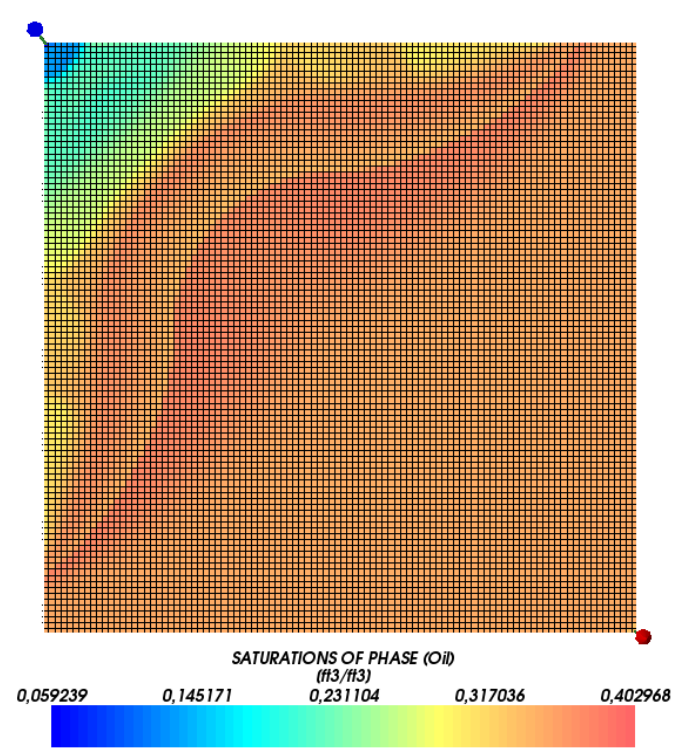

(b)

Figure 14: Oil saturation front at 100 days - Case 3. a) IMPEC; b) WATTS

A time step comparison for a 100x100 grid is shown in Fig. 15. From this figure, we can verify that the maximum time-step employed by the Watts' formulation was about five times larger than that used by the IMPEC approach. In order to see the effect of the mesh size, we present in Fig. 16 the total CPU time for three meshes. We can see that the relative performance of the Watts' formulation in comparison with the IMPEC formulation improves as the mesh is refined.

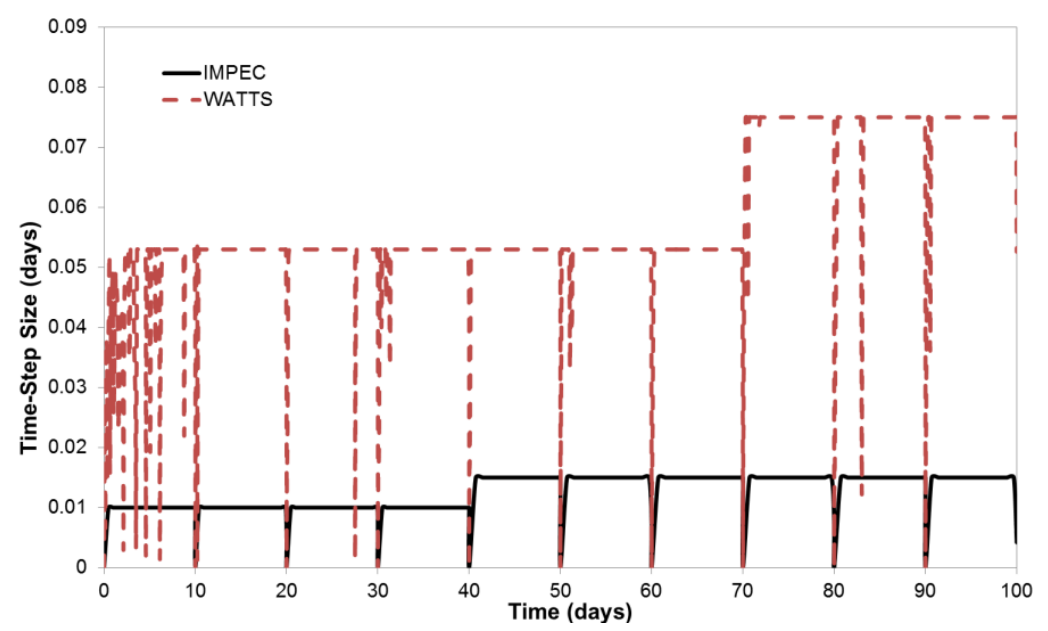

Figure 15: Time-step comparison - Case 3. 


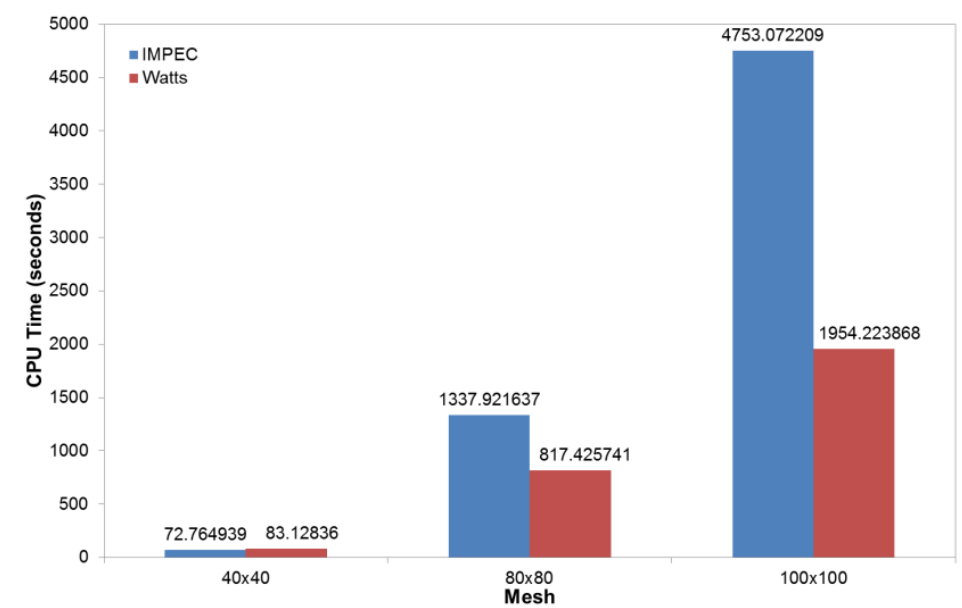

Figure 16: Effect of mesh size on the CPU time - Case 3.

Case 4 presents $\mathrm{CO}_{2}$ injection in a heterogeneous and anisotropic reservoir. The reservoir and fluid data used for this case are shown in Tables 7 and 8. The absolute permeability in the $x$ and $z$ directions are shown in Fig. 17. The same permeability as in the $x$ direction was used for the y direction.

Table 7: Reservoir data - Case 4.

\begin{tabular}{ccc}
\hline Property & Value & Unit \\
Length & 800 & $\mathrm{ft}$ \\
Width & 800 & $\mathrm{ft}$ \\
Height & 200 & $\mathrm{ft}$ \\
First Layer Depth & 0 & $\mathrm{ft}$ \\
Porosity & 0.3 & \\
Relative Permeability Model & Stone II & \\
Initial Water Saturation & 0.25 & $\mathrm{psia}$ \\
Initial Pressure & 3000 & $\mathrm{oF}$ \\
Formation Temperature & 80 & $\mathrm{MSCF} / \mathrm{d}$ \\
Injector Rate & 20000 & $\mathrm{psia}$ \\
Producer BHP & 3000 & \\
Grid & $20 \times 20 \times 5$ &
\end{tabular}

Table 8: Component data - Case 4.

\begin{tabular}{ccc}
\hline Component & Initial Reservoir Composition & Injection Fluid Composition \\
\hline $\mathrm{CO} 2$ & 0.0100 & 0.95 \\
$\mathrm{C} 1$ & 0.1900 & 0.05 \\
$\mathrm{NC} 16$ & 0.8000 & \\
\hline
\end{tabular}




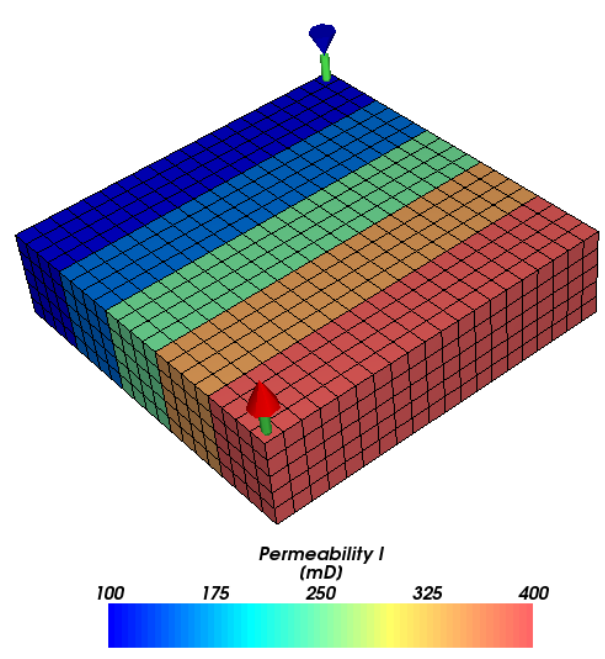

(a)

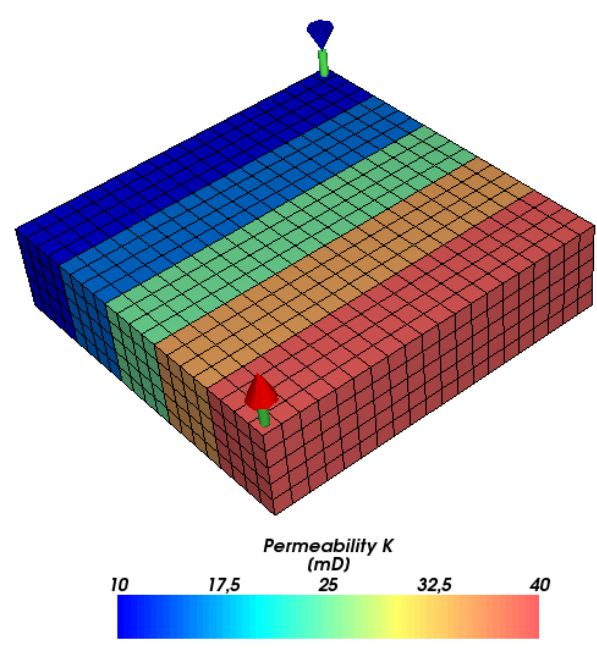

(b)

Figure 17: Absolute permeability. a) x direction; b) z direction.

Figures 18 and 19 present the oil and gas volumetric rates, respectively. Figure 20 shows the gas saturation front at 500 days. From these figures, we can observe a very good match between both formulations.

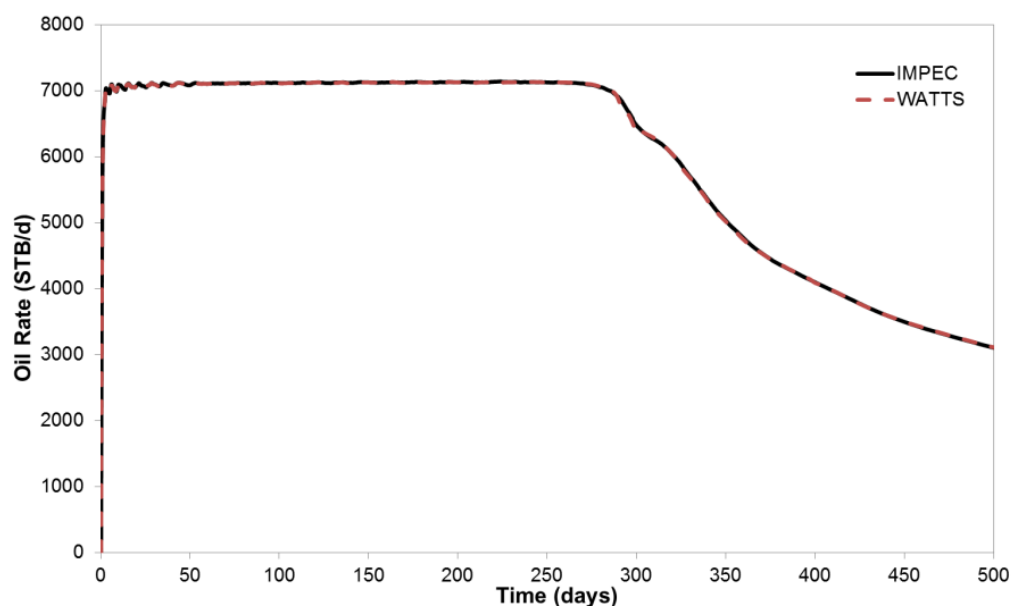

Figure 18: Oil volumetric rate - Case 4.

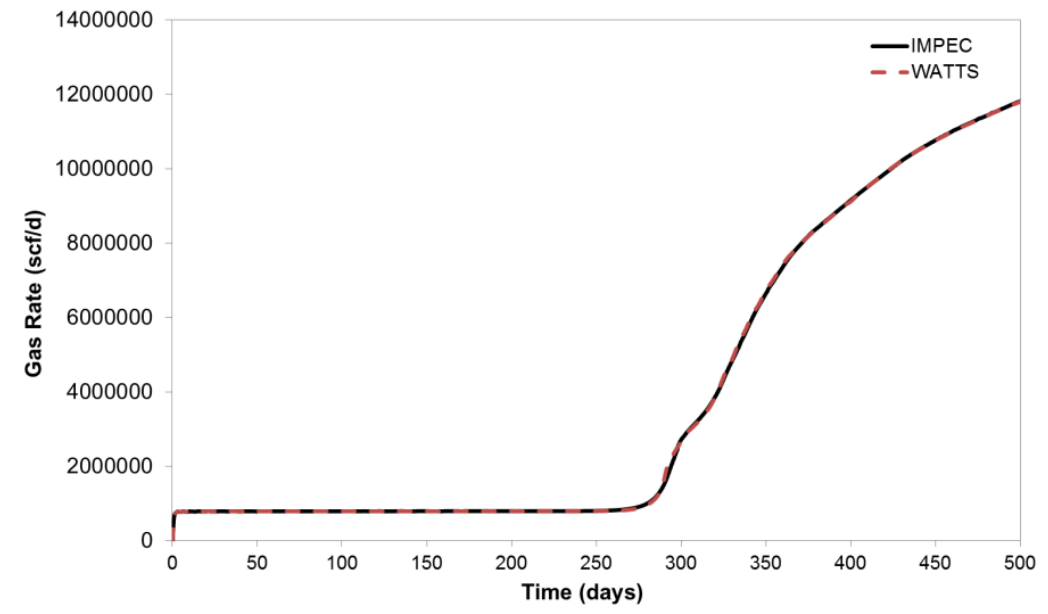

Figure 19: Gas volumetric rate - Case 4. 


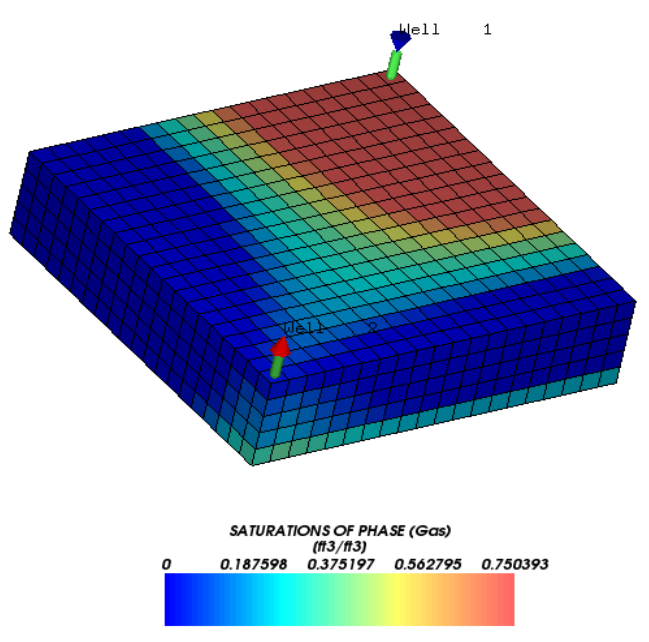

(a)

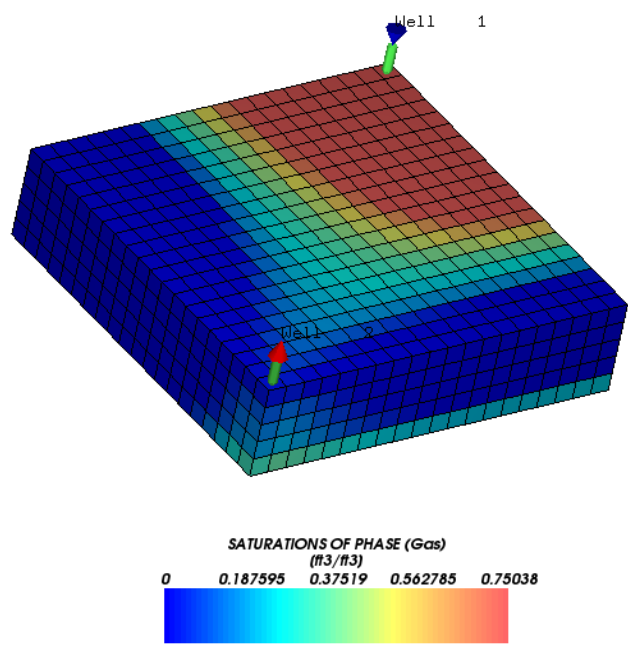

(b)

Figure 20: Gas saturation front - Case 4. a) IMPEC; b) Watts.

Figure 21 presents the time-steps history used by both formulations. From this figure, we can observe that on average the time-step employed by the Watts' formulation was eight times larger than that of the IMPEC formulation. Table 9 presents the average CPU employed by both formulations. We can infer from the table that Watts' formulation was about 2.3 times faster than the IMPEC formulation.

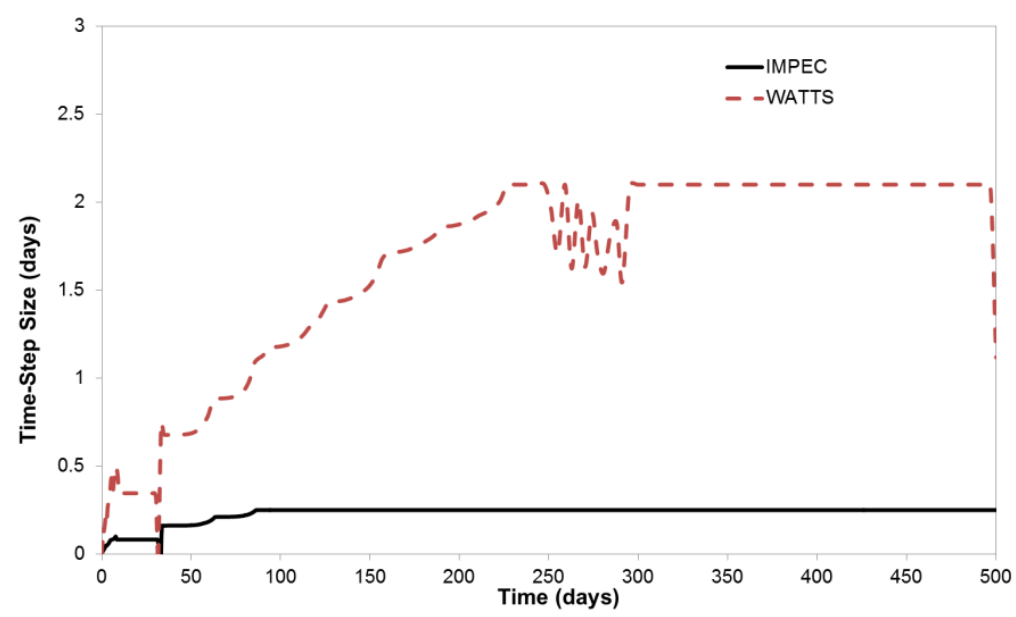

Figure 21: Time-step comparison - Case 4.

Table 9: CPU Time comparison - Case 4.

\begin{tabular}{ccc}
\hline Formulation & CPU time & Speed-up ratio \\
\hline IMPEC & 84.58 & 1 \\
Watts & 36.59 & 2.33 \\
\hline
\end{tabular}

\section{CONCLUSIONS}

In this work, we implemented the Watts' formulation for reservoirs using Cartesian grids into the UTCOMP simulator. Also, two interpolation functions for evaluating the 
physical properties at each interface of the control volume were implemented: UDS (upwind difference scheme) and a third-order TVD method. For most of the case studies tested, the Watts' formulation implemented in this work was around two times faster than the original IMPEC formulation of the UTCOMP simulator. The next step in this research is to implement the Watts' formulation for unstructured grids using the EbFVM (element-based finite-volume method).

\section{Acknowledgments}

The authors would like to acknowledge Abu Dhabi National Oil Company for the financial support for this work.

\section{REFERENCES}

[1] Watts, J. W., "A Compositional Formulation Based on Pressure and Saturation Equations.” SPE Reservoir Engineering Journal 1, N 4, 243-252, 1986.

[2] Ács, G., Doleschall, S., and Farkas, E., "General Purpose Compositional Model." Soc. Pet. Eng. J. 25, $\mathrm{N}^{\mathrm{o}}$ 4, 543-553, 1985.

[3] Chang, Y. B., "Development and Application of An Equation of State Compositional Simulator." (PhD. Thesis) Department of Petroleum and Geosystems Engineering, The University of Texas at Austin, 1990.

[4] Spillette, A. G., Hillestad, J. G., and Stone, H. L., "A High-Stability Sequential Solution Approach to Reservoir Simulation." Fall Meeting of the Society of Petroleum Engineers of AIME, Las Vegas, Nevada, 1973.

[5] Branco, C. M. and Rodriguez, F., "A Semi-Implicit Formulation for Compositional Reservoir Simulation.” SPE Advanced Technology Series 4, Nº 1, 171-177, 1996.

[6] Chang, Y. B., Pope, G. A., and Sepehrnoori, K., "A higher-order finite-difference compositional simulator." Journal of Petroleum Science and Engineering 5, $\mathrm{N}^{\mathrm{o}}$ 1, 35-50, 1990.

[7] Runbin, B. and Blunt, M. J., "Higher-Order Implicit Flux Limiting Schemes for Black Oil Simulation." $11^{\text {th }}$ SPE Symposium on Reservoir Simulation, USA, 219-229, 1991. 